\title{
OBJETOS DE APRENDIZAGEM, COMPETÊNCIAS PROFISSIONAIS PARA PROFISSIONAIS DE SAÚDE E E-LEARNING: ESTUDOS PARA DESENVOLVIMENTO DE UMA TAXONOMIA
}

\author{
Learning Objects, Professional Competencies for Health Professional and e-Learning: \\ Main Elements for Developing a Taxonomy
}

\author{
Lina Barreto Brasil'; Mary Caroline Skelton-Macedo²; Francisco Eduardo Campos³; Ana Estela Haddad ${ }^{4}$
}

\begin{abstract}
Resumo A Universidade Aberta do SUS - UNA-SUS (www.unasus.gov.br) foi concebida pelo Ministério da Saúde do Brasil, em parceria com 18 universidades públicas e serviços estaduais e municipais de saúde, com o objetivo de oferecer capacitação e educação profissional aos trabalhadores da saúde em atividade no SUS, o Sistema Único de Saúde, que conta, hoje, com cerca de 2,5 milhões de profissionais da área. Um dos principais focos da UNA-SUS é a produção de objetos de aprendizagem (OAs) interoperacionais e reutilizáveis, segundo as demandas identificadas pelos profissionais em atividade, tanto pelos que lidam com atenção à saúde, quanto pelos que lidam com assuntos de gerenciamento. Os OAs são publicados em arquivo gratuito, aberto ao público (http://ares.unasus.gov.br/acervo/). Um assunto estratégico para a expansão e, concomitantemente, para a manutenção da qualidade do que é sustentado com recursos públicos, é o estabelecimento de padrões. Para esse fim, planejamos construir uma taxonomia capaz de definir as competências pedagógicas, técnicas e profissionais, bem como as habilidades desejáveis que se espera de um determinado $\mathrm{OA}$, para que este seja interoperacional e reutilizável.
\end{abstract}

Palavras-chave: Objetos de Aprendizagem, e-Learning, Tecnologias da Informação e Comunicação, Atenção Primária à Saúde, Odontologia.

Abstract The Open University of the Unified Health System - UNA SUS (www.unasus.gov.br) was developed by the Brazilian Ministry of Health, in partnership with 18 public universities, as well as state and municipal health services, in order to provide capacity building and education for health professionals working at Brazilian Unified Health System (SUS), which has approximately 2.5 million health workers. One of its main components is the production of interoperable and reusable learning objects (LOs) according to the needs identified by the professionals at work, those who deal with healthcare, as well as management matters. The LOs are published in an archive that is open to the public and free of charge (http://ares.unasus.gov.br/acervo/). One strategic issue for expanding, and at the same time maintaining quality of what is financed by public funds, is establishing standards, and for this purpose we plan to develop a taxonomy capable of describing pedagogical, technological and professionals competencies and skills that are expected to be developed by a determined $\mathrm{LO}$, in order for it to be interoperable and reusable.

Keywords: Learning Objects, e-Learning, Information and Communication Technologies (ICT), Primary Health Care, Dentistry.

\footnotetext{
1. Doutoranda do Programa de Ciências Odontológicas da Faculdade de Odontologia da USP, Consultora da Universidade Aberta do SUS - UNASUS, Fiocruz, Ministério da Saúde; 2. Professora Doutora de Teleodontologia, Pesquisadora e Coordenadora Adjunta do Núcleo de Teleodontologia e Professora Colaboradora do Programa de Pós Graduação em Ciências Odontológicas da Faculdade de Odontologia da USP; 3. Professor Titular de Medicina Preventiva da Faculdade de Medicina e Coordenador do NESCON da UFMG, Secretário Executivo da UNASUS, Fellow da Global Health Workforce Alliance, WHO; 4. Professora Associada, Pesquisadora do Núcleo de Teleodontologia e Professora do Programa de Pós Graduação em Ciências Odontológicas da Faculdade de Odontologia da USP.
} 


\section{Introdução e Objetivos}

A Universidade Aberta do Sistema Único de Saúde - UNA-SUS - foi lançada pelo Ministério da Saúde em 2008, visando a fomentar a expansão de uma rede de integração entre instituições de educação superior que compõem o sistema educacional público e o SUS funcionando por meio do intercâmbio de experiências, compartilhamento de material educacional, cooperação para desenvolvimento e implementação de novas tecnologias educacionais em saúde. A UNA-SUS apoia o processo de aprendizagem em serviço e certifica os alunos egressos dos cursos que oferece.

Dessa forma é possível levar a cada trabalhador de saúde oportunidades de aprendizado, tais como material autoinstrucional, cursos livres e de atualização, cursos de aperfeiçoamento, especialização e até mesmo mestrados profissionais. O uso da estratégia de educação a distância (EAD) online permite que os profissionais da saúde aprendam de acordo com suas necessidades, no seu tempo e no local que lhe for mais conveniente.

A estratégia adotada pela UNA-SUS para ampliar a capacidade de produção dos recursos educacionais (Objetos de Aprendizagem - OA digitais) pelas universidades públicas que compõem a sua rede foi instituir padrões para os objetos de aprendizagem, como reutilização, interoperabilidade e foco nas necessidades de aprendizagem profissional dos trabalhadores, permitindo assim uma maior efetividade dos programas educacionais. Para apoiar o armazenamento e acesso público e gratuito a esses recursos educacionais, a UNA-SUS construiu um acervo aberto, público e gratuito de recursos especialmente voltados para a saúde (http://ares.unasus.gov.br/acervo/).

A produção de OAs para atender a grande demanda de capacitação (i.e: o Ministério da Saúde solicita uma média de 10 novos temas ou recursos educacionais/mês à UNA-SUS), exige velocidade de produção sem perda de qualidade. Porém, apesar dos grandes avanços observados nos recursos educacionais produzidos desde 2008, as 18 IES que integram a rede UNA-SUS - número em crescimento - ainda não possuem equipes com expertise suficiente na produção desse tipo de recurso que thes possibilite o atendimento das demandas mencionadas.

Os resultados disso podem ser constatados por uma busca rápida no ARES (o acervo citado anteriormente) por recursos educacionais reutilizáveis, interoperáveis e alinhados com as necessidades de aprendizagem dos profissionais aos quais se destinam, como mostra o gráfico da Fig. 1 a seguir:

\section{Objetos de aprendizagem, competências profissionais} em sáúde e em EAD - estudos para uma taxonomia

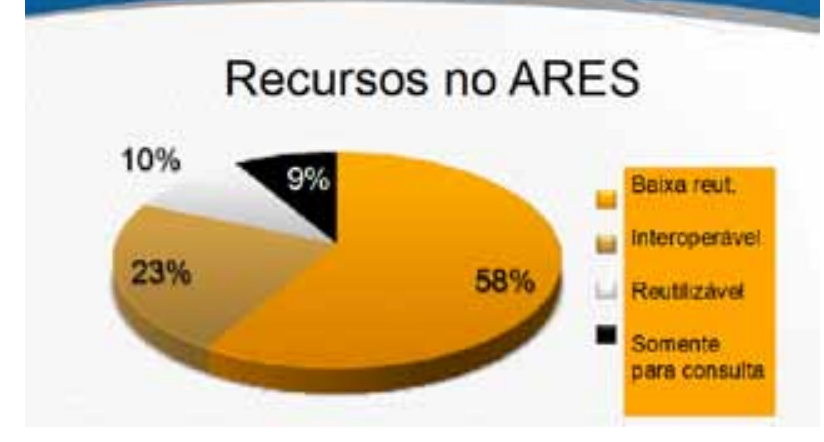

Figura 1: Características dos Recursos Educacionais armazenados no ARES, BRASIL, L. S.B, 2012.

Os padrões para os recursos educacionais indicados pela UNA-SUS são os seguintes:

- Interoperáveis - utilizam padrões tecnológicos que Ihes conferem características que lhes permitem trafegar em múltiplas plataformas de forma transparente para os usuários;

- Reutilizáveis - os recursos são desenvolvidos para terem alta granularidade e baixo nível de agregação;

- Atendimento das necessidades de aprendizagem dos alunos - os recursos devem ser desenvolvidos para atender as necessidades de aprendizagem dos profissionais da saúde, ou seja, devem basearse em objetivos de aprendizagem em lugar de em objetivos de ensino. 
Por nossas observações de campo e acompanhamento do trabalho das diversas instituições produtoras da rede UNA-SUS desde 2008, uma das maiores dificuldades na construção dos recursos educacionais é o alinhamento desses recursos com as necessidades de aprendizagem dos alunos. Para facilitar esse alinhamento, a proposta desta pesquisa é subsidiar a construção de uma taxonomia das propriedades pedagógicas de um objeto de aprendizagem digital a partir dos seguintes elementos:

1. Alinhamento entre as competências dos profissionais de saúde (no âmbito desta pesquisa será analisado apenas o conjunto de competências dos dentistas que atuam no programa de saúde da família) e o objeto de aprendizagem a ser desenvolvido;

2. Alinhamento entre o projeto instrucional e o objeto de aprendizagem ${ }^{1}$;

3. Alinhamento entre os objetos a serem desenvolvidos e as competências da equipe da EAD, incluindo as competências em EAD.

O objetivo desta pesquisa é desenvolver uma Taxonomia que descreva e relacione as características pedagógicas, tecnológicas e as competências profissionais típicas envolvidas na capacitação em serviço online promovida pela Universidade Aberta do SUS - UNASUS -, que auxilie as Universidades que compõem a rede UNA-SUS a imprimir aos recursos educacionais desenvolvidos as qualidades da reutilização, da interoperabilidade e do alinhamento da ação educacional com as necessidades do profissional/serviço de saúde para os quais foi desenvolvida ${ }^{2,6,8}$.

\section{Metodologia}

O O contexto educacional neste trabalho de pesquisa considera que o processo de educação para os profis- sionais do SUS deve ser focado no desenvolvimento de competências, o qual é centrado na aprendizagem dos participantes, para sua atuação como pessoas, como profissionais e como cidadãos ${ }^{4}$.

Segundo Durand $(1998)^{5}$, a aprendizagem é entendida como o processo psicológico pelo qual o indivíduo adquire as competências para o trabalho. A competência refere-se à capacidade de realizar algo e para isso, é necessário possuir recursos e utilizá-los em determinada situação, com o objetivo de obter resultados. Esses recursos são os conhecimentos, as habilidades e as atitudes, adquiridos via formação e experiências de vida. Ver Fig. 2 a seguir.

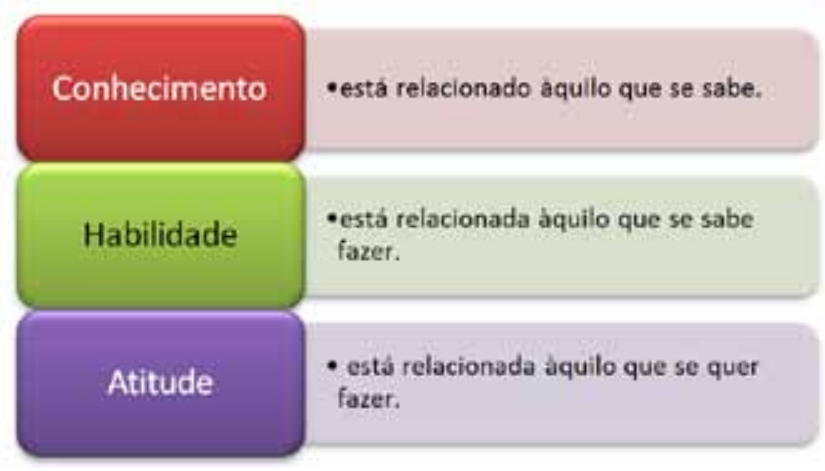

Figura 2: Os três conceitos básicos da formação profissional, BARRETO, L.S.B, 2012.

Serão analisados e sistematizados os estudos sobre as principais características desejáveis para que os OAs da UNA-SUS sejam efetivos em seus propósitos educacionais e reutilizáveis, tais como: competências profissionais versus projeto instrucional do OA, i.e: pesquisas sobre as competências profissionais e recursos educacionais em repositórios; reusabilidade e interoperabilidade; alinhamento entre o projeto instrucional e o objeto de aprendizagem; competências de equipes de educação a distância. Essa pesquisa utilizará como sujeitos os dentistas que atuam no programa de governo de saúde da família para os quais já existem competências profissionais bem definidas e que estejam inseridos em ações educacionais da UNA-SUS. A partir dessa análise, será proposta uma taxonomia relacionando competências profissionais, 
reusabilidade e integração ensino/serviço envolvidas na construção de objetos digitais de aprendizagem para trabalhadores dentistas da saúde de acordo com os padrões sugeridos pela UNA-SUS.

A pesquisa utilizará metodologia voltada para identificar as principais questões do estudo, os stakeholders, o referencial teórico do estudo, os instrumentos de pesquisa mais adequados a cada abordagem. Apresentamos a seguir as três metodologias selecionadas para esta pesquisa:

\section{Estudo}

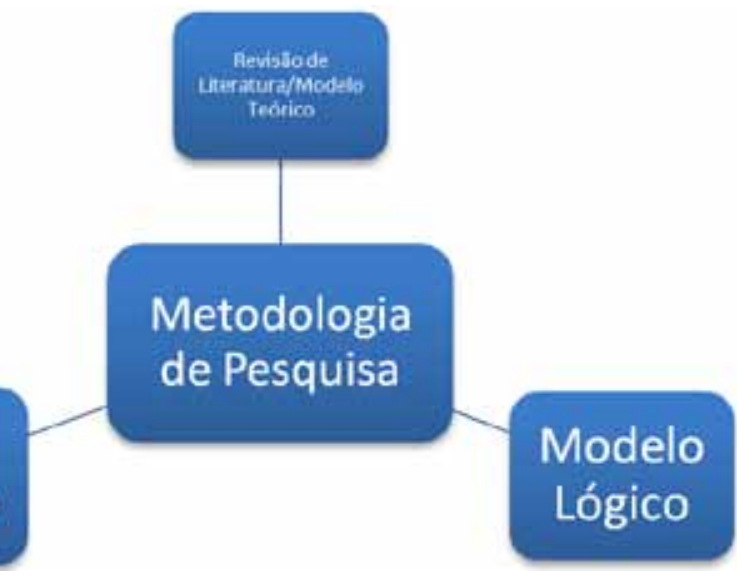

\section{Papel de Cada Metodologia:}

1. Modelo Lógico: análise da situação atual; seleção de um problema fundamental; identificação dos interessados na pesquisa (stakeholders); análise dos problemas, suas causas e efeitos; identificação dos objetivos; planejamento da pesquisa.

\section{Componentes do Modelo Lógico:}

1.a) Matriz Lógica: compreende uma análise da situação atual; a seleção de um problema fundamental; identificação dos objetivos;

1.b) Arvore dos Interessados: a identificação dos interessados na pesquisa;

1.c) Árvore de Problemas (causa e efeito): uma análise dos problemas e suas causas e efeitos;

2. Estudo de Caso: descrever o contexto da UNA-SUS no qual as características de reusabilidade, interoperabilidade e integração ensino/serviço foram definidas como padrão dos recursos educacionais a serem desenvolvidos pela rede UNA-SUS; analisar os resultados dessas definições no contexto da produção dos recursos educacionais frente às demandas feitas pelo Ministério da Saúde à UNA-SUS; apontar evidências da importância destes padrões para o maior reuso, a maior circulação e a maior qualidade na produção dos recursos educacionais que circulam na rede UNA-SUS. Ou seja, segundo Yin, $2000^{16}$, o estudo de caso vai ajudar a saber o "como"e o "porquê" do objeto da pesquisa.

\section{Componentes do Estudo de Caso:}

2.a) Questões do Estudo - definir qual a melhor estratégia para realizar a pesquisa (a forma da questão: quem, o que, onde, como, por que);

2.b) Proposições de estudo - focar a atenção sobre os diversos aspectos envolvidos no escopo da pesquisa;

2.c) Unidade(s) de análise(s) - definição do próprio caso;

2.d) Definir a lógica que une os dados às proposições;

2.e) Definir os critérios para se interpretar as descobertas do estudo.

3. Revisão Literatura - apoiar a construção de um modelo teórico que vai direcionar o estudo de caso.

\section{Papel da Revisão de Literatura:}

Elaboração de uma teoria preliminar relacionada ao tópico de estudo, anterior à realização de coleta de dados, que irá orientar toda a pesquisa, especialmente o estudo de caso.

\section{Resultados Esperados}

Em seu artigo “The Post-LEGO Learning Object”, Wiley (1999) ${ }^{14}$ compara duas metáforas usadas para explicar o conceito da reusabilidade dos recursos educacionais/ OAs, quais sejam: 
- A metáfora do LEGO muito utilizada para explicar de forma fácil como os recursos/OAs (diversos pequenos pedaços de uma ação educacional que contêm significado educacional em si mesmos) podem se conectar a qualquer outros OAs, por qualquer pessoa, formando assim novas ações educacionais versus

- A metáfora do ÁTOMO, proposta por Wiley para substituir a do LEGO, tendo em vista que o átomo também é um pequeno pedaço que pode ser combinado com outros átomos formando novos átomos diferentes, mas que, ao contrário do LEGO, um ÁTOMO não pode ser combinado com qualquer outro átomo, pois essa combinação depende da estrutura de cada átomo e precisa de algum treinamento para ser realizada.

Pela leitura do artigo, podemos entender que a ideia defendida por Wiley (que foi um dos idealizadores do conceito de objetos de aprendizagem como recursos educacionais reutilizáveis por excelência) é que a reutilização dos recursos educacionais depende completamente da sua estrutura instrucional/pedagógica, ou seja, do porquê, para que e para quem foram criados.

O problema, implícito na metáfora, é a necessidade de expertise técnica para realizar a construção dos OAs (átomos) e posterior combinação deles com outros OAs.
A proposta desta pesquisa é justamente facilitar o processo de construção desses recursos educacionais pelas Universidades, que precisam ser alinhados com as necessidades de aprendizagem dos alunos e devem desenvolver as competências profissionais necessárias que os auxiliem a responder aos problemas com que se defrontam em seu cotidiano do serviço.

Para isso, definiu-se a criação de uma Taxonomia como instrumento de apoio da produção desses recursos pelas Universidades parceiras integrantes da rede UNA-SUS. Na Ciência da Informação, taxonomia é um sistema utilizado para classificar e facilitar o acesso à informação. Seu objetivo é representar conceitos através de termos; melhorar a comunicação entre especialistas e outros públicos; propor formas de controle da diversificação e oferecer um mapa do processo de conhecimento. É, portanto, um vocabulário controlado de uma determinada área do conhecimento e um instrumento que permite alocar, recuperar e comunicar informações dentro de um sistema.

A estruturação de uma taxonomia demanda a organização de conceitos em processos que incluem categorização e classificação de conceitos, definição das relações entre esses conceitos e tratamento da terminologia empregada nos conceitos e relações da estrutura. Na Tab. 1 a seguir, são apresentadas algumas das principais características/utilidades dessa taxonomia em construção.

\begin{tabular}{|c|c|}
\hline Auxílio na Organização da Informação & Gestão da inforn \\
\hline $\begin{array}{l}\text { Agiliza a comunicação } \\
\text { Auxilia na organização e compartilhamento do conhecimento } \\
\text { Auxilia os usuários quanto aos termos específicos e mais adequados para } \\
\text { Produção do OA } \\
\text { Atribui responsabilidades de avaliação, organização, eliminação e arquivamento } \\
\text { Facilita a busca nos acervos } \\
\text { Facilita o acesso à informação } \\
\text { Metodologia de organização, recuperação, representação e disponibilização. } \\
\text { Permite a reusabilidade da informação } \\
\text { Permite acesso imediato à informação } \\
\text { Permite agregação de dados } \\
\text { Permite alocar, recuperar e comunicar informações dentro de um sistema } \\
\text { Permite melhor interpretação da informação } \\
\text { Permite o usuário nomear e compartilhar informações } \\
\text { Permite uma melhor integração da informação } \\
\text { Usada para localizar as informações relevantes }\end{array}$ & $\begin{array}{l}\text { Mapa de área que s } \\
\text { Parte importante da } \\
\text { Pilar da Gestão da Ir } \\
\text { Regras de alto nível }\end{array}$ \\
\hline
\end{tabular}

Tabela 1: Usos, Aplicações e Finalidades da Taxonomia

Fonte: Elementos Constitutivos do Conceito de Autonomia, AGANETTE. E. ALVARENGA. L. e SOUZA. R.R. (2010) 
O resultado que se espera dessa pesquisa é uma Taxonomia que descreva e relacione as características pedagógicas, tecnológicas e as competências típicas envolvidas na capacitação em serviço de dentistas, que auxilie a imprimir aos OAs desenvolvidos as qualidades da reutilização, da interoperabilidade e do alinhamento da ação educacional com as necessidades do profissional/serviço de saúde para os quais foi desenvolvida.

\section{Referências}

1. A.D. Wiley, Connecting learning objects to instructional design theory: A definition, a metaphor, and a taxonomy, The Edumetrics Institute, Logan, UT, 2010.

2. B. Blackburn Taxonomy design types. AlIM E-doc Magazine, Maryland, USA. v.20, n.3, p.14-16, maio/jun. 2006.

3. B. S. Bloom et al. (eds.). Taxionomia de objetivos educacionais. Porto Alegre: Globo, 1972. V.1.

4. Competências profissionais para egressos e temas abordados nos Cursos de Especialização em Saúde da Família da UnA-SUS, MIMEO, DAB/MS, Brazil, 2010

5. DURAND. Forms of incompetence. Trabalho apresentado na 'Conference of Management of Competence', Oslo, 1998
6. D. L. Silva, R. R. Souza and M. B. Almeida Comparação de metodologias para construção de ontologias e vocabulários controlados. Anais do Seminário de pesquisa em ontologia no Brasil, Niterói, 2008, Niterói.

7. M.C.C.Baranauskas, H.V.Rocha, M.C. Martins and J.V.V.d'Abreu Uma taxonomia para ambientes de aprendizado baseados no computador, artigo do livro O computador na sociedade do conhecimento, Campinas, SP, 1999.

8. R.R. Relvão Estudo Sobre a Utilização e Interoperabilidade entre Conteúdos de Aprendizagem com Diferentes Granularidades, Master thesis, Universidade do Minho, Minho, 2006.

9. Terra, J.C.C.; et al. Taxonomia: elemento fundamental para a gestão do conhecimento. 2005. Disponível em : http://www.terraforum.com.br Acesso em: 30 dez. 2013.

10. Wiley, D. A. \& Nelson, L. M. (1998). The fundamental object [On-line]. Available: http://wiley.ed.usu.edu/docs/fundamental.html

11. Wiley, D. A. (1999). Learning objects and the new CAl: So what do I do with a learning object? [On-line]. Available: http://wiley.ed.usu.edu/docs/instruct-arch.pdf

12. Wiley, D. A., South, J. B., Bassett, J., Nelson, L. M., Seawright, L. L., Peterson, T., \& Monson, D. W. (1999). Three common properties of efficient online instructional support systems. The ALN Magazine, 3 (2)[On-line]. Available: http://www.aln.org/alnweb/ magazine/Vol3_issue2/wiley.htm

13. Wiley, D. A. (2000). Learning object design and sequencing theory . Unpublished doctoral dissertation, Brigham Young University . Available: http://davidwiley.com/ papers/dissertation/dissertation.pdf

14. Wiley, D. A. (2000). The Post-LEGO Learning ObjectAvailable: http://cmap. upb.edu.co/rid=1146063082796_1162237027_9479/The\%20Post-LEGO\%20 Learning\%200bject_wiley.pdf

15. Wiley, D. A. (2013) Website David Wiley Available: http://davidwiley.org/

16. Yin, R. K. (2001) Estudo de caso: planejamento e métodosBookman, Porto Alegre. 